\title{
The eccentric universe
}

\author{
Arjun Berera*, Roman V. Buniy† and \\ Thomas W. Kephart \\ * School of Physics, University of Edinburgh, Edinburgh, EH9 3JZ, United \\ Kingdom \\ † Institute of Theoretical Science, University of Oregon, Eugene, OR 97403, USA \\ $\ddagger$ Department of Physics and Astronomy, Vanderbilt University, Nashville, TN \\ 37235, USA \\ E-mail: ab@ph.ed.ac.uk, roman@uoregon.edu and \\ thomas.w.kephart@vanderbilt.edu
}

\begin{abstract}
For a universe containing cosmological constant together with uniform arrangements of magnetic fields, strings, or domain walls, exact solutions to the Einstein equations are shown to lead to a universe with ellipsoidal expansion. The magnetic field case is the easiest to motivate and has the highest possibility of finding application in observational cosmology.

PACS numbers: 98.80.-k, 98.62.En, 04.40.Nr
\end{abstract}

\section{Introduction}

The first year WMAP results $[1,2,3]$ contain interesting large-scale features which warrant further attention $[4,5]$. First, there is the suppression of power at large angular scales $\left(\theta \gtrsim 60^{\circ}\right)$, which is reflected most distinctly in the reduction of the quadrupole $C_{2}$. This effect was also seen in the COBE results $[6,7]$. However, WMAP was able to make more precise measurements of $C_{2}$. Next, the quadrupole $C_{2}$ and octupole $C_{3}$ are found to be aligned. In particular, the $\ell=2$ and 3 powers are found to be concentrated in a plane inclined about $30^{\circ}$ to the Galactic plane. In a coordinate system in which the equator is in this plane, the $\ell=2$ and 3 powers are primarily in the $m= \pm \ell$ modes. The axis of this system defines a unique direction and supports an idea of power in one direction being suppressed compared to the power in the orthogonal plane. These effects seem to suggest one (longitudinal) direction may have expanded differently from the other two (transverse) directions, where the transverse directions describe the equatorial plane mentioned above.

After the COBE experiment, Monte Carlo studies were used [8] to cast doubt on quadrupole suppression and analyses of the WMAP data $[1,2,3,4,5]$ have arrived at similar conclusions. Nevertheless, interesting physical effects are not ruled out and a realistic physical model that could explain some or all effects in the WMAP data would be of interest.

It would be most satisfying to explain global anisotropy in the universe by a simple modification of the conventional Friedman-Robertson-Walker (FRW) model. As a first step in this direction, we will consider an energy-momentum tensor which is spatially non-spherical or spontaneously becomes non-spherical. Such situations could occur 
when defects or magnetic fields are present. Magnetic fields [9] and cosmic defects [10] can arise in various ways. Moreover, large scale magnetic fields exist in the universe, perhaps up to cosmological scales $[9,11]$. These considerations motivate us to examine the effect magnetic fields and defects have on the expansion of the universe [12].

To begin to understand the form, significance and implications of an asymmetric universe, we here modify the standard spherically symmetric FRW cosmology to a form with only planar symmetry [13]. Our choice of energy-momentum will result in non-spherical expansion from a spherical symmetric initial configuration: an initial co-moving sphere will evolve into an ellipsoid that can be either prolate or oblate depending on the choice of matter content. We then explore the effects of this type of asymmetric expansion on modes of a multipole expansion. For the sake of clarity, we first give some general properties of cosmologies with planar symmetry (The universe looks the same from all points but they all have a preferred direction.) and then investigate one case in detail. Our exemplar will be a universe filled with uniform magnetic fields and cosmological constant. This is perhaps the most easily motivated, analytically solvable case to consider and it will give us a context in which to couch the discussion of other examples with planar symmetry and cases where planar symmetry is broken.

To set the stage, consider an early epoch in the universe at the onset of cosmic inflation, where strong magnetic fields have been produced in a phase transition $[14,15,16,17,18]$. The magnitude of the magnetic field and vacuum energy ( $\Lambda$ ) densities initially are about the same, but eventually $\Lambda$ dominates. It was estimated [16] that the initial magnetic field energy produced in the electroweak phase transition was within an order of magnitude of the critical density. Other phase transitions may have even higher initial field values $[17,18]$. Hence, it is not unphysical to consider a universe with B-fields and $\Lambda$ of comparable magnitudes. If the B-fields are aligned in domains, then some degree of inflation is sufficient to push all but one domain outside the horizon. (Below we also discuss cases where there are still a few domains within the horizon.)

\section{Planar symmetric cosmology}

To make the simplest directionally anisotropic universe, we modify spherical symmetry of FRW space-time into planar symmetry. (Cylindrical symmetry is, of course, not appropriate since it introduces a preferred location of the axis of symmetry.) The most general form of planar-symmetric metric (up to a conformal transformation) is [13] $\left(g_{\mu \nu}\right)=\operatorname{diag}\left(1,-e^{2 a},-e^{2 a},-e^{2 b}\right)$, where $a$ and $b$ are functions of $t$ and $z$; and the $x y$-plane is the plane of symmetry. We also impose translational symmetry along the $z$-axis; the functions $a$ and $b$ now depend only on $t$. For the above metric, the nonzero-components of the Ricci tensor then are

$$
\begin{aligned}
& R_{0}^{0}=-\left(2 \ddot{a}+\ddot{b}+2 \dot{a}^{2}+\dot{b}^{2}\right), \\
& R^{1}{ }_{1}=R^{2}{ }_{2}=-\left(\ddot{a}+2 \dot{a}^{2}+\dot{a} \dot{b}\right), \\
& R^{3}{ }_{3}=-\left(\ddot{b}+\dot{b}^{2}+2 \dot{a} \dot{b}\right) .
\end{aligned}
$$

To support the symmetry of space-time, the energy momentum tensor for the matter has to have the same symmetry:

$$
\left(T^{\mu}{ }_{\nu}\right)=(8 \pi G)^{-1} \operatorname{diag}(\xi, \eta, \eta, \zeta) .
$$


Here the energy density $\xi$, transverse $\eta$ and longitudinal $\zeta$ tension densities are functions of time only. Examples of plane-symmetric spaces include space uniformly filled with either magnetic fields, static aligned strings, or static stacked walls; i.e., the defects are at rest with respect to the cosmic background frame. Of course, we can add to these any spherically-symmetric contributions: vacuum energy, matter, radiation (see Table 1). The entries in Table 1 are scaled according to Eq. (1); for instance, $\lambda=8 \pi G \Lambda$.

Table 1. Various contributions to the components of the energy-momentum tensor in Eq. (1).

\begin{tabular}{lccc}
\hline & $\xi$ & $\eta$ & $\zeta$ \\
\hline vacuum energy & $\lambda$ & $\lambda$ & $\lambda$ \\
matter & $\rho$ & 0 & 0 \\
radiation & $\rho$ & $-\frac{1}{3} \rho$ & $-\frac{1}{3} \rho$ \\
magnetic field & $\epsilon$ & $-\epsilon$ & $\epsilon$ \\
strings & $\epsilon$ & 0 & $\epsilon$ \\
walls & $\epsilon$ & $\epsilon$ & 0 \\
\hline
\end{tabular}

The Einstein equations corresponding to Eq. (1) are

$$
\begin{aligned}
& \dot{a}^{2}+2 \dot{a} \dot{b}=\xi, \\
& \ddot{a}+\ddot{b}+\dot{a}^{2}+\dot{a} \dot{b}+\dot{b}^{2}=\eta, \\
& 2 \ddot{a}+3 \dot{a}^{2}=\zeta .
\end{aligned}
$$

In addition, we need the equation expressing covariant conservation of the energymomentum [a direct consequence of Eqs. (2)-(4)]:

$$
\dot{\xi}+2 \dot{a}(\xi-\eta)+\dot{b}(\xi-\zeta)=0 .
$$

Before considering specific models for the energy-momentum, we first establish several general features of an anisotropic universe described by Eqs. (2)-(5). We assume that before anisotropic effects became important, the universe was isotropic [19], $a_{\mathrm{i}}=b_{\mathrm{i}}$, and was expanding isotropically, $\dot{a}_{\mathrm{i}}=\dot{b}_{\mathrm{i}}>0$. Without loss of generality we set $a_{\mathrm{i}}=0$, which is equivalent to setting the initial scale factor to unity. During the initial phase of anisotropic expansion when non-uniform contributions to the energy-momentum are more significant, different tension densities in the longitudinal and transverse directions cause the universe to expand non-spherically. At a later time, when all contributions except for the vacuum energy fade away, longitudinal and transverse expansion rates become equal and the expansion again proceeds isotropically. Thus, an initial co-moving sphere evolves into an ellipsoid; whether it is oblate $(a>b)$ or prolate $(a<b)$ depends on which tension dominated during the initial phase of deformation.

For all known forms of classical matter, the components of the energy-momentum tensor satisfy the dominant energy condition [20]; in our case it says $\xi \geq 0, \xi \geq \eta$, and $\xi \geq \zeta$ (see Table 1 for examples). These conditions lead to various bounds and asymptotics of which we list here a few important examples.

First, one can show that for the initial conditions given above, the energy density never increases. Also, the space always expands transversally, and unless the magnetic 
field contribution dominates, the space expands longitudinally. Next, the transverse expansion rate has its maximum at $t=t_{\mathrm{i}}$ and the same is true for the longitudinal expansion rate unless the wall contribution dominates.

Second, the eccentricity [21] is bounded. Indeed, Eqs. (2) and (4) lead to $\dot{a}-\dot{b}=(\zeta-\xi-2 \ddot{a}) / 2 \dot{a}$, which upon using the energy condition $\xi \geq \zeta$ gives the bound $e^{a-b} \leq \dot{a}_{\mathrm{i}} / \dot{a}$. Solving Eq. (4) asymptotically for large $t$, we find $\dot{a} \sim(\zeta / 3)^{\frac{1}{2}}$, which leads to the asymptotic bound

$$
e^{a-b} \lesssim\left(\xi_{\mathrm{i}} / \zeta\right)^{\frac{1}{2}}
$$

Note that in an expanding universe $\xi_{\mathrm{i}} \geq \xi \geq \zeta$ and so (6) does not forbid $a>b$. A sub-case with vacuum energy and any combination of magnetic fields and strings aligned in the same direction has $\xi=\zeta$, and similarly we find

$$
e^{a-b} \sim\left(\xi_{\mathrm{i}} / \zeta\right)^{\frac{1}{2}}
$$

Third, let us expand Eqs. (5) for large $t$ to find

$$
(\delta \xi)^{\cdot}+n\left(\frac{1}{3} \lambda\right)^{\frac{1}{2}} \delta \xi=0
$$

here $n=3-2 \delta \eta / \delta \xi-\delta \zeta / \delta \xi$. For cosmological constant plus magnetic field, strings, or walls, $n=4,2$ or 1 , respectively. Thus the energy density approaches its asymptotic value $\lambda$ according to $\delta \xi \propto e^{-t / t_{\xi}}$ with characteristic time $t_{\xi}=n^{-1}(3 / \lambda)^{\frac{1}{2}}$.

We consider here three exactly solvable cases: cosmological constant plus either uniform magnetic field, homogeneously aligned strings, or homogeneously stacked walls. We concentrate on the case of magnetic fields, which is the easiest to motivate.

\section{Magnetic field}

In the case of magnetic fields, conservation of energy-momentum, Eq. (5), has a simple form: $\dot{\epsilon}+4 \dot{a} \epsilon=0$. Solving this equation for $\dot{a}$ and substituting the result into Eq. (4), we arrive at the equation $\epsilon \ddot{\epsilon}-\frac{11}{8} \dot{\epsilon}^{2}+2 \epsilon^{2}(\lambda+\epsilon)=0$ whose general solution is given implicitly by

$$
t-t_{\mathrm{i}}=\frac{1}{4} \int_{\epsilon}^{\epsilon} \epsilon_{\mathrm{i}} d \epsilon\left(\frac{1}{3} \lambda \epsilon^{2}+\frac{4}{3} \epsilon_{\mathrm{i}}^{\frac{1}{4}} \epsilon^{\frac{11}{4}}-\epsilon^{3}\right)^{-\frac{1}{2}} .
$$

Once the function $\epsilon$ is found from Eq. (9), the functions $a$ and $b$ are found from Eqs. (2) and (5) as follows:

$$
\begin{aligned}
& a=\frac{1}{4} \ln \left(\epsilon_{\mathrm{i}} / \epsilon\right), \\
& b=\frac{1}{2} \ln \frac{\lambda+4 \epsilon_{\mathrm{i}}\left(\epsilon / \epsilon_{\mathrm{i}}\right)^{\frac{3}{4}}-3 \epsilon}{\lambda+\epsilon_{\mathrm{i}}}-\frac{1}{4} \ln \left(\epsilon / \epsilon_{\mathrm{i}}\right) .
\end{aligned}
$$

To find the behavior of $a$ and $b$ for large $t$ we need to find asymptotics for the integral in Eq. (9). The integral diverges for small $\epsilon$ (which corresponds to large $t$ ), and so we extract the divergent part first; this results in $t-t_{\mathrm{i}} \approx \frac{1}{4}(3 / \lambda)^{\frac{1}{2}} \ln \left(\epsilon_{\mathrm{i}} / \epsilon\right)-\tau$, where

$$
\tau=\frac{1}{4} \int_{0}^{\epsilon_{\mathrm{i}}} d \epsilon\left[\left(\frac{1}{3} \lambda \epsilon^{2}\right)^{-\frac{1}{2}}-\left(\frac{1}{3} \lambda \epsilon^{2}+\frac{4}{3} \epsilon_{\mathrm{i}}^{\frac{1}{4}} \epsilon^{\frac{11}{4}}-\epsilon^{3}\right)^{-\frac{1}{2}}\right] .
$$

Large $t$ asymptotics give

$$
\begin{aligned}
& \epsilon \sim \epsilon_{\mathrm{i}} e^{-4(\lambda / 3)^{\frac{1}{2}}\left(t-t_{\mathrm{i}}+\tau\right)}, \\
& a \sim(\lambda / 3)^{\frac{1}{2}}\left(t-t_{\mathrm{i}}+\tau\right), \\
& b \sim(\lambda / 3)^{\frac{1}{2}}\left(t-t_{\mathrm{i}}+\tau\right)-\frac{1}{2} \ln \left(1+\epsilon_{\mathrm{i}} / \lambda\right) .
\end{aligned}
$$


Eqs. (13) and (14) lead to an oblate expansion with $e^{a-b} \sim\left(1+\epsilon_{\mathrm{i}} / \lambda\right)^{\frac{1}{2}}$, which agrees with the general result found in Eq. (7).

We can include matter and still find exact solutions. For example, for a universe filled with cosmological constant, magnetic fields and dust with density $\rho$, we find

$$
\rho=\rho_{\mathrm{i}}\left(\epsilon / \epsilon_{\mathrm{i}}\right)^{\frac{3}{4}}\left[1+F\left(\epsilon / \epsilon_{\mathrm{i}}\right)\right]^{-1}\left[\frac{\lambda+\left(\rho_{\mathrm{i}}+4 \epsilon_{\mathrm{i}}\right)\left(\epsilon / \epsilon_{\mathrm{i}}\right)^{\frac{3}{4}}-3 \epsilon}{\lambda+\rho_{\mathrm{i}}+\epsilon_{\mathrm{i}}}\right]^{-\frac{1}{2}},
$$

where

$$
\begin{aligned}
F\left(\epsilon / \epsilon_{\mathrm{i}}\right)= & \frac{3}{8}\left(\rho_{\mathrm{i}} / \epsilon_{\mathrm{i}}\right)\left[1+\left(\lambda+\rho_{\mathrm{i}}\right) / \epsilon_{\mathrm{i}}\right]^{\frac{1}{2}} \\
& \int_{\epsilon / \epsilon_{\mathrm{i}}}^{1} \mathrm{~d} x x^{-\frac{1}{4}}\left[\lambda / \epsilon_{\mathrm{i}}+\left(4+\rho_{\mathrm{i}} / \epsilon_{\mathrm{i}}\right) x^{\frac{3}{4}}-3 x\right]^{-\frac{3}{2}} .
\end{aligned}
$$

Equation (10) is unchanged but Eq. (11) becomes

$$
\begin{aligned}
b & =\frac{1}{2} \ln \frac{\lambda+\left(\rho_{\mathrm{i}}+4 \epsilon_{\mathrm{i}}\right)\left(\epsilon / \epsilon_{\mathrm{i}}\right)^{\frac{3}{4}}-3 \epsilon}{\lambda+\rho_{\mathrm{i}}+\epsilon_{\mathrm{i}}} \\
& -\frac{1}{4} \ln \left(\epsilon / \epsilon_{\mathrm{i}}\right)+\frac{1}{2} \ln \left[1+F\left(\epsilon / \epsilon_{\mathrm{i}}\right)\right],
\end{aligned}
$$

and time dependence of the above functions $\rho(\epsilon), a(\epsilon)$ and $b(\epsilon)$ can be deduced from the function $\epsilon(t)$, which is now given implicitly by

$$
t-t_{\mathrm{i}}=\frac{1}{4} \int_{\epsilon}^{\epsilon_{\mathrm{i}}} \mathrm{d} \epsilon\left[\frac{1}{3} \lambda \epsilon^{2}+\frac{1}{3} \epsilon_{\mathrm{i}}^{-\frac{3}{4}}\left(4 \epsilon_{\mathrm{i}}+\rho_{\mathrm{i}}\right) \epsilon^{\frac{11}{4}}-\epsilon^{3}\right]^{-\frac{1}{2}} .
$$

\section{Strings and Walls}

For strings, solutions to the Einstein equations can be obtained similarly to the magnetic field case. Here we give only large $t$ behavior of these solutions:

$$
\begin{aligned}
\epsilon & \sim \epsilon_{\mathrm{i}} e^{-2(\lambda / 3)^{\frac{1}{2}}\left(t-t_{\mathrm{i}}+\tau\right)}, \\
a & \sim(\lambda / 3)^{\frac{1}{2}}\left(t-t_{\mathrm{i}}+\tau\right), \\
b & \sim(\lambda / 3)^{\frac{1}{2}}\left(t-t_{\mathrm{i}}+\tau\right)-\frac{1}{2} \ln \left(1+\epsilon_{\mathrm{i}} / \lambda\right),
\end{aligned}
$$

where

$$
\tau=\frac{1}{2} \int_{0}^{\epsilon} \mathrm{i}_{\mathrm{i}} d \epsilon\left[\left(\frac{1}{3} \lambda \epsilon^{2}\right)^{-\frac{1}{2}}-\left(\frac{1}{3} \lambda \epsilon^{2}-\frac{2}{3} \epsilon_{\mathrm{i}}^{-\frac{1}{2}} \epsilon^{\frac{7}{2}}+\epsilon^{3}\right)^{-\frac{1}{2}}\right] .
$$

As in the case of magnetic field, $e^{a-b} \sim\left(1+\epsilon_{\mathrm{i}} / \lambda\right)^{\frac{1}{2}}$, in agreement with Eq. (7).

Solving the case of walls proceeds in a different fashion. Asymptotic forms of the corresponding solutions read

$$
\begin{aligned}
\epsilon & \sim \epsilon_{\mathrm{i}} e^{-(\lambda / 3)^{\frac{1}{2}}\left(t-t_{\mathrm{i}}\right)}\left[\frac{(1-\sigma)^{\frac{1}{3}}}{1+\sigma}+\frac{6 \sigma F}{(1-\sigma)^{2}}\right]^{-1}, \\
a & \sim(\lambda / 3)^{\frac{1}{2}}\left(t-t_{\mathrm{i}}\right)-\frac{2}{3} \ln (1-\sigma), \\
b & \sim(\lambda / 3)^{\frac{1}{2}}\left(t-t_{\mathrm{i}}\right)+\ln \left[\frac{(1-\sigma)^{\frac{1}{3}}}{1+\sigma}+\frac{6 \sigma F}{(1-\sigma)^{2}}\right],
\end{aligned}
$$




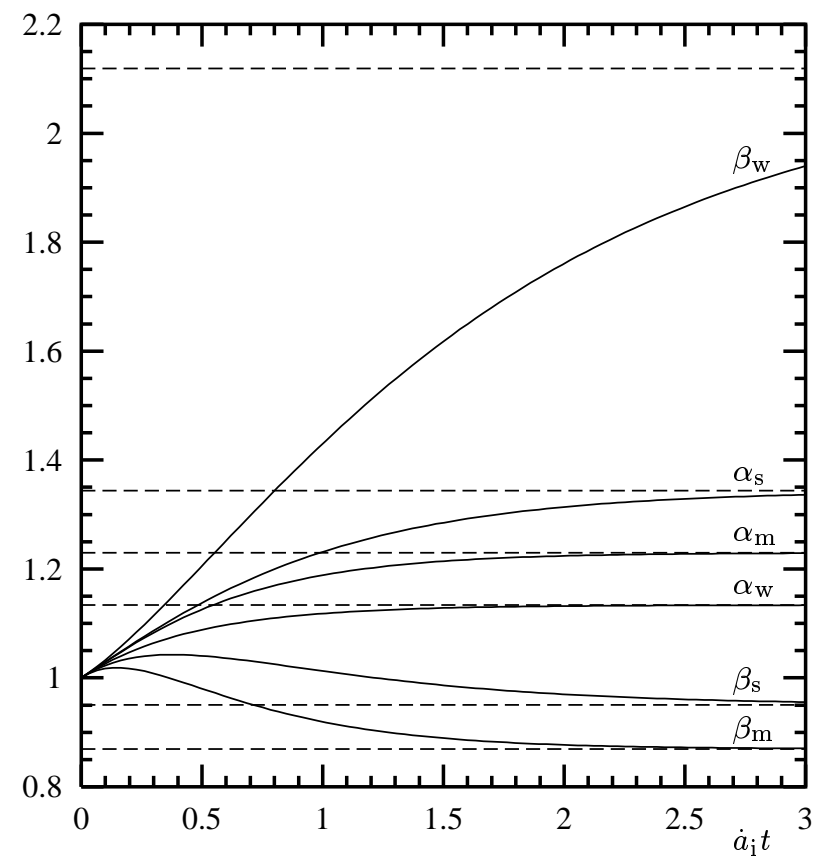

Figure 1. The scale factors $\alpha=e^{a-c}$ and $\beta=e^{b-c}$, where $c=\left(\frac{1}{3} \lambda\right)^{\frac{1}{2}}\left(t-t_{\mathrm{i}}\right)$ corresponds to the model with only cosmological constant. For the cases of magnetic field, strings, and walls, the same initial conditions are used. Solid lines are exact solutions and dashed lines are asymptotics for large $t$.

where

$$
\sigma=\frac{\left(1+\epsilon_{\mathrm{i}} / \lambda\right)^{\frac{1}{2}}-1}{\left(1+\epsilon_{\mathrm{i}} / \lambda\right)^{\frac{1}{2}}+1}
$$

and

$$
F=\int_{1}^{\infty} d x \frac{\left(x^{3}-\sigma\right)^{\frac{4}{3}}}{\left(x^{3}+\sigma\right)^{2}}
$$

One can derive the bound $F \geq \frac{1}{3}(1-\sigma)^{\frac{4}{3}}(1+\sigma)^{-1}$, which leads to a prolate expansion with $e^{a-b} \lesssim 1$.

In Fig. 1 we compare the scale factors for the cases of magnetic field, strings, and walls. Since in all three cases the functions $a$ and $b$ asymptotically approach the corresponding solution for the model with only cosmological constant, $c=$ $\left(\frac{1}{3} \lambda\right)^{\frac{1}{2}}\left(t-t_{\mathrm{i}}\right)$, this term is conveniently factored out of the scale factors. Fig. 2 similarly compares the corresponding energy densities. In both figures we used the same initial conditions for the three cases. These conditions are fully specified by the value $\epsilon_{\mathrm{i}} / \lambda$; we chose $\epsilon_{\mathrm{i}}=\lambda$ which leads to $\dot{a}_{\mathrm{i}}=\left(\frac{2}{3} \lambda\right)^{\frac{1}{2}}$.

\section{Conclusions}

Since the time of COBE, various speculations have been put forward to explain the origin of the suppressed quadrupole. One idea has been to explore topological features 


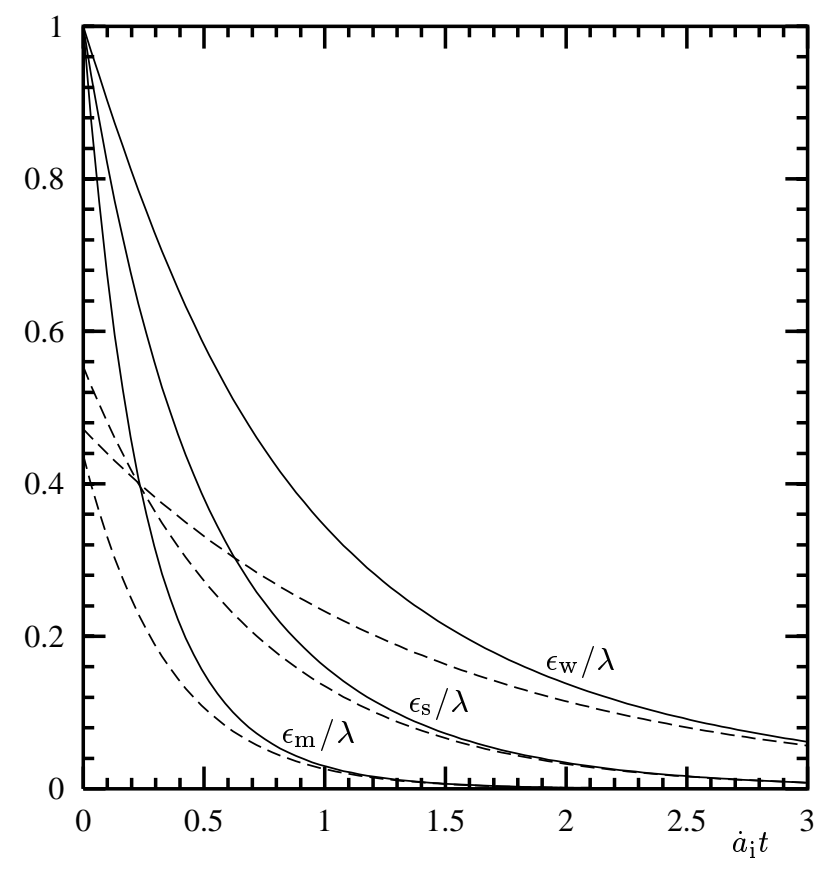

Figure 2. The ratio $\epsilon / \lambda$ for the same three cases and initial conditions as in Fig. 1.

with specific emphasis on the "small universe" picture with a toroidal $T^{3}$ topology [22]. Another idea has been to study the possibility of a small inflation, which somehow gets tuned to about the scale of the present-day Hubble radius [8, 23, 24]. However, very little has been suggested for physical models that could explain the new features suggested by the WMAP data. An obvious modification of the $T^{3}$-model would be to make one dimension smaller than the other two and get a $T_{a}^{2} \times T_{b}^{1}$ asymmetric toroidal topology [5]. Other topologies could also work. There are many choices [26, 25] and the results could be profound.

In this paper we have not departed radically from conventional cosmology, but rather considered a simple modification of the FRW model. We determined how magnetic fields, strings, and walls affect the expansion rate of the universe. Before solving the Einstein equations we found the asymptotic values and bounds for the ratio of longitudinal and transverse sizes for several interesting cases. Next, we presented exact analytical solutions for a universe filled with magnetic field and gave asymptotic results for the case of defects. Finally, we mentioned particle physics motivated models that could yield the sorts of magnetic fields and/or defects of importance for asymmetric expansion. We have found that a universe filled with aligned B-fields and $\Lambda$ expands non-spherically: a co-moving sphere at $t_{\mathrm{i}}$ evolves into an ellipsoid. The eccentricity is calculable and fixed by the initial conditions. Aside from being potentially applicable to explaining some features in the WMAP data, the models and the results presented in this paper differ from those in the already mentioned Ref. [12]. The first of the papers in Ref. [12] examined the situation where there is a uniform magnetic field and no cosmological constant. By adding cosmological constant, we have found the exact solution for the model which is physically relevant. The second 
paper in Ref. [12] treats only small anisotropies and determines only asymptotic large time behavior. Our calculations apply to anisotropy of arbitrary strength and we have obtained the exact solutions to the Einstein equations which are valid at all times.

The effects of non-spherical expansion and small inflation are potentially measurable depending on the degree of eccentricity and expansion attained and the quality of data. In realistic particle physics motivated models, where the B-field in question is associated with particular gauge fields and the cosmological constant $\Lambda$ arises from some specific inflaton model, additional predictions may emerge. For example, a strongly aligned B-field at the beginning of inflation may affect density perturbations in a measurable way [27], perhaps through polarization effects, and a small inflation that follows may mean these perturbations are observable at the largest scales today. We have provided only homogeneous solutions. To apply our results to structure formation and CMB data, the next step will be to begin an analysis of linear density perturbations in such a Universe.

The model developed here can be extended to cases of less symmetric evolution. Some examples are models with an aligned B-field plus cosmic strings neither aligned parallel nor perpendicular to this field, or models with various kinds of misaligned cosmic defects. Another possibility with more physical motivation is a model with B-fields and $\Lambda$ where there are still a few misaligned magnetic domains within the horizon at the end of inflation. While this model is more complicated, it may also be more realistic as the initial domain size is more in line with the models of cosmological phase transitions $[14,15,16,17,18]$. Finally, while there could be other combinations of cosmological constant, magnetic fields, defects, and geometry that arise in brane world physics and produce asymmetric expansion, we believe it is important to have considered the simplest well motivated case first.

\section{Acknowledgments}

We thank Gary Hinshaw for stimulating discussions of the WMAP data. The work of $\mathrm{AB}$ was supported by PPARC, and the work of RVB and TWK was supported by U.S. DoE grant number DE-FG05-85ER40226.

\section{References}

[1] C. L. Bennett et al., Astrophys. J. Suppl. 148 (2003) 1.

[2] D. N. Spergel et al., Astrophys. J. Suppl. 148 (2003) 175.

[3] G. Hinshaw et al., Astrophys. J. Suppl. 148 (2003) 135.

[4] M. Tegmark, A. de Oliveira-Costa and A. Hamilton, arXiv:astro-ph/0302496.

[5] A. de Oliveira-Costa, M. Tegmark, M. Zaldarriaga and A. Hamilton, arXiv:astro-ph/0307282.

[6] G. F. Smoot et al., Astrophys. J. 396 (1992) L1.

[7] A. Kogut et. al, arXiv:astro-ph/9601060.

[8] A. Berera, L. Z. Fang and G. Hinshaw, Phys. Rev. D 57 (1998) 2207.

[9] P. P. Kronberg, Rept. Prog. Phys. 57 (1994) 325.

[10] M. B. Hindmarsh and T. W. Kibble, Rept. Prog. Phys. 58 (1995) 477.

[11] S. D. Wick, T. W. Kephart, T. J. Weiler and P. L. Biermann, Astropart. Phys. 18 (2003) 663.

[12] A cosmological model with uniform magnetic field only (no vacuum energy as in the present work) was solved in G. Rosen, Phys. Rev. 136, (1964) B297. (Also see Y. B. Zeldovich and I. D. Novikov, Relativistic Astrophysics. Vol. 2, The University of Chicago Press, 1983.) Asymptotics for models with various small anisotropies were obtained in J. D. Barrow, Phys. Rev. D 55 (1997) 7451.

[13] A. H. Taub, Annals Math. 53 (1951) 472.

[14] G. K. Savvidy, Phys. Lett. B 71 (1977) 133.

[15] S. G. Matinian and G. K. Savvidy, Nucl. Phys. B 134 (1978) 539. 
[16] T. Vachaspati, Phys. Lett. B 265 (1991) 258.

[17] K. Enqvist and P. Olesen, Phys. Lett. B 329 (1994) 195.

[18] A. Berera, T. W. Kephart and S. D. Wick, Phys. Rev. D 59 (1999) 043510.

[19] The subscript "i" refers to the moment of transition from isotropic to anisotropic dynamics.

[20] S. W. Hawking and G. F. R. Ellis, The Large Scale Structure of Space-Time, Cambridge University Press, 1973.

[21] To simplify formulas, we write results in terms of the ratio of scale factors $e^{a} / e^{b}$, which can be easily expressed in terms of the true eccentricity.

[22] G. F. R. Ellis, Gen. Relativ. Gravit. 2 (1971) 7.

[23] J. M. Cline, P. Crotty and J. Lesgourgues, JCAP 0309 (2003) 010.

[24] M. Bastero-Gil, K. Freese and L. Mersini-Houghton, arXiv:hep-ph/0306289.

[25] J. G. Ratcliffe and S. T. Tschantz, Class. Quant. Grav. 15 (1998) 2613.

[26] G. W. Gibbons, Nucl. Phys. B 472 (1996) 683.

[27] C. Tsagas and R. Maartens, Phys. Rev. D 61 (2000) 083519. 